
\title{
28 Research Square \\ Clinical Characteristics of SARS-CoV-2 Infection in Haikou: a study of a family cluster
}

\author{
Hui Juan Chen \\ Hainan General Hospital \\ Jie Qiu \\ Hainan General Hospital \\ Biao Wu \\ Hainan General Hospital \\ Zhen Ping Wang \\ Hainan General Hospital \\ Yang Chen \\ Hainan General Hospital \\ Feng Chen ( $\sim$ fenger0802@163.com ) \\ Hainan General Hospital
}

\section{Research}

Keywords: sever acute respiratory syndrome coronavirus 2(SARS-CoV-2), clinical characteristics, family cluster, pneumonia

Posted Date: April 29th, 2020

DOI: https://doi.org/10.21203/rs.3.rs-23245/v1

License: (c) (i) This work is licensed under a Creative Commons Attribution 4.0 International License.

Read Full License 


\section{Abstract}

Background: The outbreak of sever acute respiratory syndrome coronavirus 2(SARS-CoV-2) has become a great threat to the world. No study has been done on the mild or asymptomatic SARS-CoV-2 in a family cluster.

Methods: We report the epidemiological, clinical, laboratory, radiological, and clinical outcomes of five patients in a family cluster.

Results: We enrolled a family of five patients who was confirmed with SARS-CoV-2 infection. One of them worked in Wuhan and returned to Danzhou, Hainan on January 22,2020. The other four family members, who did not travel to Wuhan, became infected with the virus after several days of contact with the family member. Five family members (aged 33-57years) presented with fever, cough or no symptom onset. Three of them had negative nucleic test on first swab sampling. One of them was not confirmed until the third nucleic acid test. Two of them had radiological ground-glass lung opacities. Two patients presenting with fever had lymphopenia or decreased white blood cells. No one had increased C-reactive protein or lactate dehydrogenase levels. After treatment, they were discharged.

Conclusions: Person-to-person transmission of SARS-CoV-2 was confirmed in family setting. Concerns should be raised for the asymptomatic persons in a family cluster.

\section{Introduction}

The unexplained cases of pneumonia were first announced by the Health Commission of Hubei province, China on Dec 31, 2019. The pneumonia was confirmed to be caused by a novel enveloped RNA betacoronavirus 2 that has currently been renamed as severe acute respiratory syndrome coronavirus 2 (SARS-CoV-2), which shares similarity to SARS-CoV in gene.

The SARS-CoV-2 was regarded as a public health emergency of international concern by the World Health Organization (WHO) on January 31,2020. On March 11,2020, the WHO declared that SARS-CoV-2 can be characterized as a pandemic. As of March 16, 2020, a total of 167,511 laboratory-confirmed cases had been reported globally. The SARS-CoV-2 has resulted 6606 deaths by March 16 . The total number of cases and deaths outside China has overtaken the total number of cases in China. Many researches [16] had been done on the clinical and radiological characteristics in SARS-CoV-2. In consideration of the outbreak of SARS-CoV-2, it is of great significance to understand the implications of possible transmission from mild or asymptomatic SARS-CoV-2 infected cases. To date, few studies had reported regarding the mild or asymptomatic SARS-CoV-2 in family. In this study, we describe the epidemiological, clinical, radiological, laboratory, and clinical outcomes of a family cluster of five patients in Hainan, one of whom had a history of working in Wuhan, and the other family members had never been to Wuhan or other places in Hubei.

\section{Methods}


From January to February 2020, we investigated 5 confirmed cases of familial clustered SARS-CoV-2 infection admitted to in Hainan General Hospital. Among them, three of the family members was asymptomatic on admission.

\section{Data Collection}

This study was waved by the institutional review board of the Hainan General Hospital. Written informed consents of all participants were obtained before the study. We collected and analyzed the history, epidemiological, biochemical, radiological data of all these cases. Briefly, data regarding on symptoms and disease onset, hospitalization, treatment, laboratory tests, and clinical outcomes was analyzed according to the medical records. The computed tomography (CT) images were reviewed by two experienced radiologists.

\section{SARS-CoV-2 infection confirmation}

SARS-CoV-2 infection was confirmed by repeated twice positive results in throat swabs or respiratory specimens of real-time reverse transcription polymerase chain reaction (RT-PCR) assay with SARS-CoV-2 nucleic acid detection kits as described in the previous study[5]. Throat swab samples of patients who were suspected of SARS-CoV-2 infection were collected for extracting SARS-CoV-2 RNA. After collection, these samples were put into a collection tube, and total RNA was extracted within 2 hours using the respiratory sample RNA isolation kit. Same procedures were performed as described in previous study[5]. The suspension was used for real-time reverse transcription polymerase chain reaction (RT-PCR) assay of SARS-CoV-2 RNA. Two target genes, including open reading frame $1 \mathrm{ab}$ (ORF1ab) and nucleocapsid protein $(\mathrm{N})$, were simultaneously amplified and tested during the real-time RT-PCR assay. Target 1 (ORF1ab): forward primer CCCTGTGGGTTTTACACTTAA; reverse primer ACGATTGTGCATCAGCTGA; and the probe 5'-FAM-CCGTCTGCGGTATGTGGAAAGGTTATGG-BHQ1-3'. Target 2 (N): forward primer GGGGAACTTCTCCTGCTAGAAT; reverse primer CAGACATTTTGCTCTCAAGCTG; and the probe 5'-FAMTTGCTGCTGCTTGACAGATT-TAMRA-3'. The realtime RT-PCR assay was performed using a 2019-nCoV nucleic acid detection kit according to the manufacturer's protocol (Shanghai bio-germ Medical Technology Co Ltd). RT-PCR assay was performed under the following conditions: incubation at $50{ }^{\circ} \mathrm{C}$ for 15 minutes and $95^{\circ} \mathrm{C}$ for 5 minutes, 40 cycles of denaturation at $94^{\circ} \mathrm{C}$ for 15 seconds, and extending and collecting fluorescence signal at $55^{\circ} \mathrm{C}$ for 45 seconds. A cycle threshold value (Ct-value) less than 37 was defined as a positive test result, and a Ct-value of 40 or more was defined as a negative test. These diagnostic criteria were based on the recommendation by the National Institute for Viral Disease Control and Prevention (China) (http://ivdc.chinacdc.cn/kyjz/202001/t20200121_211337. html). A medium load, defined as a Ct-value of 37 to less than 40 , required confirmation by retesting.

\section{Clinical Features Of Subjects Positive For Sars-cov-2}

\section{Clinical features of subjects positive for SARS-CoV-2}


Patient 1 (father) flew from Wuhan to Haikou on January 22, 2020. He had no history of contact with animals, visits to markets including the Huanan seafood wholesale market in Wuhan, or eating game meat in restaurants. After returning Hainan, he lived with his family including his wife (Patient 2) and three sons (Patient 3,4 ). Patient 5 (aunt of patient3,4) did not contact with patient 1 but have close contact with the rest of the family. Patient 1 presented with fever, cough and fatigue the next day he went home (Jan 23, 2020). He was confirmed with COVID-19 infection on January 23, 2020. Patients 2-5 stayed at home for quarantine. Relatives 2-4 was diagnosed with SARS-CoV-2 infection 14,17 and 16 days after close contact with patient 1 . All of these patients did not present with any clinical symptoms at the time of confirmation. Patient 2 reported fatigue three days after diagnosis. Patient three and four reported cough and diarrhea, respectively on February 9,2020. Patient 5 developed with fever, chest tightness and shortness of breath 14 days after close contact with the relatives (patient 2-4). She was confirmed with SARS-CoV-2 infection on February 6,2020. The incubation period was estimated to be around 14 days.

Of the five patients, three were male and two were female. The age ranged from 33-57 years (Table 1 ). Only patient 5 had one comorbidity of cardiovascular disease and two had history of fever. The older patients (aged $>55$ years: patient 1 and 2 ) developed generalized weakness. Patient 4 had cough. Patients 3 and 4 were younger adults and one of them had diarrhea. They are smokers. Patient 5 also presented with pleuritic chest pain and shortness of breath. Except for patient 5, all four had normal total white blood cell counts and neutrophil cell count. Patient 1 had lymphopenia. Patient 2-5 had normal Creactive protein, lactate dehydrogenase levels and activated thromboplastin time. All five patients underwent CT examinations at their hospitalization. CT images of patient 2 and 4 did not have significantly positive findings. Patient 1 had negative CT findings on admission. Ten days after symptom onset, small patchy ground glass was observed in the upper lobe of left lung. Patient 3 and patient 5 showed multifocal patchy ground glass opacities and subsegmental areas of consolidation (Figure $2 \mathrm{~A}$, D). These lesions were gradually absorbed with the treatment (Figure 2 B, C; D, E). No other concomitant radiological abnormalities including pleural effusion, lymphadenopathy, or pneumomediastinum were seen.

\section{Treatment and Prognosis}

Patient 1 received interferon, antiviral treatment and antibiotic therapy (Moxifloxacin for 14 days). His nasopharyngeal swabs on February 3 and February 5, 2020 testing was negative. He was discharged well from hospital after 14 days hospitalization. Patient 2 was treated with antiviral (for 9 days) and antibiotic therapy (Moxifloxacin for 2 days). She had negative swabs tests on February 11 and 16 . She was discharge from hospital on February 17. Patient 3 received antiviral treatment for 6 days and Moxifloxacin for 7 days. He had negative swabs tests on February 15 and 17. He was discharged on February 18,2020. Patient 4 was only treated with interferon (the first 2 days) antiviral treatment for 7 days. He had negative swabs tests on February 15 and 16. He was discharged on February 16,2020. Patient 5 received 2-days of interferon, antiviral and antibiotic treatment for 8 days. She was discharged from hospital after twice negative swabs tests on February 12 and 14. 


\section{Treatment And Prognosis}

Patient 1 received interferon, antiviral treatment and antibiotic therapy (Moxifloxacin for 14 days). His nasopharyngeal swabs on February 3 and February 5, 2020 testing was negative. He was discharged well from hospital after 14 days hospitalization. Patient 2 was treated with antiviral (for 9 days) and antibiotic therapy (Moxifloxacin for 2 days). She had negative swabs tests on February 11 and 16 . She was discharge from hospital on February 17. Patient 3 received antiviral treatment for 6 days and Moxifloxacin for 7 days. He had negative swabs tests on February 15 and 17. He was discharged on February 18,2020. Patient 4 was only treated with interferon (the first 2 days) antiviral treatment for 7 days. He had negative swabs tests on February 15 and 16. He was discharged on February 16,2020. Patient 5 received 2-days of interferon, antiviral and antibiotic treatment for 8 days. She was discharged from hospital after twice negative swabs tests on February 12 and 14.

\section{Discussion}

Our study provides further evidence for the ability of person-to-person transmission in SARS-CoV-2[2, 7]. There exist several probable scenarios of transmission. The first and most likely scenario is that patient with pneumonia (patient 1) acquired the infection from Wuhan (unknown cause) and then transmitted the virus to patient patients $2-4$ after returning to Hainan. The second scenario is that patients 2-4 acquired the infection from patient 1 and transmitted it to patient 5 who visited their home for dinner. These results indicated the ability of person-to-person transmission in SARS-CoV-2. However, what might be the possible origin of SARS-CoV-2 should be further investigated.

Our study showed that the incubation period of the SARS-CoV-2 is relatively long. Most infected patients (patients 2-4) did not present with clinical symptoms until 14 days after close contact. This stresses the importance of home quarantine for the one who had close contact with the infected person. The virus has such high infectivity that up to $83 \%$ was found in our study. Interestingly, patient 1 did not reveal groundglass opacities on CT until 10 days after symptom onset. Patient 2 and patient 4 had negative CT findings. This highlighted the importance of PCR tests in the suspected cases. Notably, patients 2-4 were afebrile at presentation to our hospital. These asymptomatic persons might serve as a possible source to propagate the outbreak. Future studies on the epidemiological significance of these asymptomatic cases should be investigated.

The symptoms of SARS-CoV-2 were relatively mild in this family cluster. The two older patients in this family presented with fever. As expected, these two patients had decreased total white blood cell or lymphocyte. Surprisingly, no increased C-reactive protein level was found in this family members. Patient 3 and patient 5 had multifocal ground-glass changes on lung CT scan consistent with the finding of typical viral pneumonia. After treatment, these lesions were partially resolved.

Interestingly, the only symptom of younger adult (patients 4) was diarrhea. It is a pity that feces sample of the patient was not collected thus we did not know whether the result for the feces. In a recent study, 
researchers had found that live virus could be detected in the virus (positive rate 29\%) [8], supporting the notion that SARS-CoV-2 may be transmitted by the fecal route. This might be the reason why the patient presented with diarrhea. Previous study also reported that $10.6 \%$ (15 of 142) of SARS patients might present with diarrhea at presentation [9]. A higher rate of patients infected with Middle East respiratory syndrome coronavirus (MERS-CoV) also have diarrhea and viral replication could be seen in faecal samples of patients with MERS $[10,11]$. In conclusion, our study indicate that diarrhea might be the only symptom for SARS-CoV-2 patient. Much concern should be paid to this kind of patient.

Three of the members had negative nucleic acid test on their first PCR test. Particularly, patient 3 was not confirmed with SARS-CoV-2 infection until the third PCR test. False negative tests had been reported in previous studies. When this occurs, a combination of repeated swab tests and CT scanning may be helpful for these suspected individuals as suggested in previous study[12].

\section{Conclusions}

In conclusion, our study further confirmed the ability of person-to-person transmission in family homes, and therefore vigilant control measures are warranted at this early stage of the epidemic. Our study also highlighted the importance of quarantine close contacts as early as possible because asymptomatic infection appears possible (as shown in three of our patients). The incubation for the mild patients may be relatively long. Two weeks or more time of quarantine is necessary.

\section{Abbreviations}

ARS-CoV-2: sever acute respiratory syndrome coronavirus 2; CT: computed tomography; RT-PCR: real-time reverse transcription polymerase chain reaction

\section{Declarations}

\section{Acknowledgements}

Not applicable.

\section{Authors' contributions}

HJC, JQ and FC designed the study and undertook most of the work. BW, ZPW, YC and FC participated in data collection and analysis. All authors have contributed to the last version of the manuscript. The authors read and approved the final manuscript.

\section{Funding}

This work was supported by the National Nature Science Foundation of China [grant number 81971602 , 81760308, 81801684, 81871346]; the Program of Hainan Association for Science and Technology Plans 
to Youth R \& D Innovation [QCXM201919]; Hainan Provincial Natural Science Foundation of China [818MS124]; the Key science and technology project of Hainan Province [grant number ZDYF2016156].

Availability of data and materials

The datasets used during the current study are available from the corresponding author on reasonable request.

Ethics approval and consent to participate

Ethics Committee of Hainan General Hospital approved this study.

Consent for publication

Not applicable.

Conflict of Interest

The authors declare that there is no conflict of interest.

\section{References}

1. Chang, Lin M, Wei L, Xie L, Zhu G, Dela Cruz CS, Sharma L: Epidemiologic and Clinical Characteristics of Novel Coronavirus Infections Involving 13 Patients Outside Wuhan, China. JAMA 2020.

2. Chen N, Zhou M, Dong X, Qu J, Gong F, Han Y, Qiu Y, Wang J, Liu Y, Wei Y, et al: Epidemiological and clinical characteristics of 99 cases of 2019 novel coronavirus pneumonia in Wuhan, China: a descriptive study. Lancet 2020, 395:507-513.

3. Guan WJ, Ni ZY, Hu Y, Liang WH, Ou CQ, He JX, Liu L, Shan H, Lei CL, Hui DSC, et al: Clinical Characteristics of Coronavirus Disease 2019 in China. N Engl J Med 2020.

4. Huang C, Wang Y, Li X, Ren L, Zhao J, Hu Y, Zhang L, Fan G, Xu J, Gu X, et al: Clinical features of patients infected with 2019 novel coronavirus in Wuhan, China. Lancet 2020.

5. Wang D, Hu B, Hu C, Zhu F, Liu X, Zhang J, Wang B, Xiang H, Cheng Z, Xiong Y, et al: Clinical Characteristics of 138 Hospitalized Patients With 2019 Novel Coronavirus-Infected Pneumonia in Wuhan, China. JAMA 2020.

6. Ai T, Yang Z, Hou H, Zhan C, Chen C, Lv W, Tao Q, Sun Z, Xia L: Correlation of Chest CT and RT-PCR Testing in Coronavirus Disease 2019 (COVID-19) in China: A Report of 1014 Cases. Radiology 2020:200642.

7. Chan JF, Yuan S, Kok KH, To KK, Chu H, Yang J, Xing F, Liu J, Yip CC, Poon RW, et al: A familial cluster of pneumonia associated with the $\mathbf{2 0 1 9}$ novel coronavirus indicating person-to-person transmission: a study of a family cluster. Lancet 2020 .

8. Wang W, Xu Y, Gao R, Lu R, Han K, Wu G, Tan W: Detection of SARS-CoV-2 in Different Types of Clinical Specimens. JAMA 2020. 
9. Cheng VC, Hung IF, Tang BS, Chu CM, Wong MM, Chan KH, Wu AK, Tse DM, Chan KS, Zheng BJ, et al: Viral replication in the nasopharynx is associated with diarrhea in patients with severe acute respiratory syndrome. Clin Infect Dis 2004, 38:467-475.

10. Chan JF, Lau SK, To KK, Cheng VC, Woo PC, Yuen KY: Middle East respiratory syndrome coronavirus: another zoonotic betacoronavirus causing SARS-like disease. Clin Microbiol Rev 2015, 28:465-522.

11. Zhou J, Li C, Zhao G, Chu H, Wang D, Yan HH, Poon VK, Wen L, Wong BH, Zhao X, et al: Human intestinal tract serves as an alternative infection route for Middle East respiratory syndrome coronavirus. Sci Adv 2017, 3:eaao4966.

12. Xie X, Zhong Z, Zhao W, Zheng C, Wang F, Liu J: Chest CT for Typical 2019-nCoV Pneumonia: Relationship to Negative RT-PCR Testing. Radiology 2020:200343.

\section{Table}

Table 1: Summary of clinical features and laboratory results of the family cluster infected with SARSCoV-2, at presentation 


\begin{tabular}{|c|c|c|c|c|c|}
\hline & Patient 1 & Patient 2 & Patient 3 & Patient 4 & Patient 5 \\
\hline \multirow[t]{2}{*}{ Relationship } & \multirow[t]{2}{*}{$\begin{array}{l}\text { Father of } \\
\text { patients } 3 \\
\text { and } 4\end{array}$} & \multirow[t]{2}{*}{$\begin{array}{l}\text { Mother of } \\
\text { patients } 3 \\
\text { and } 4\end{array}$} & \multirow[t]{2}{*}{$\begin{array}{l}\text { Son of } \\
\text { patients } 1 \\
\text { and } 2\end{array}$} & \multirow[t]{2}{*}{$\begin{array}{l}\text { Son of } \\
\text { patients } 1 \\
\text { and } 2\end{array}$} & $\begin{array}{l}\text { Aunt of } \\
\text { patients }\end{array}$ \\
\hline & & & & & 3 and 4 \\
\hline Age(years) & 56 & 57 & 33 & 36 & 49 \\
\hline Sex & Male & Female & Male & Male & Female \\
\hline Currently smoking & No & No & Yes & Yes & No \\
\hline $\begin{array}{l}\text { Chronic medical } \\
\text { illness }\end{array}$ & None & None & None & None & $\begin{array}{l}\text { Cardiovascular } \\
\text { disease }\end{array}$ \\
\hline \multicolumn{6}{|l|}{$\begin{array}{l}\text { Presenting } \\
\text { symptoms and signs }\end{array}$} \\
\hline Fever & + & - & - & - & + \\
\hline Cough & + & - & + & - & - \\
\hline General Weakness & + & + & - & - & - \\
\hline Diarrhoea & - & - & - & - & - \\
\hline Chest tightness & - & - & - & - & + \\
\hline Shortness of breath & - & - & - & - & + \\
\hline $\begin{array}{l}\text { White blood cell } \\
\text { count }(\times 10 \varangle / L)\end{array}$ & 4.3 & 5.13 & 5.89 & 4.36 & $3.3 \downarrow$ \\
\hline Neutrophil count & 2.8 & 2.78 & 3.24 & 2.54 & $1.72 \downarrow$ \\
\hline Lymphocyte count & $0.8 \downarrow$ & 1.85 & 2.12 & 1.36 & 1.15 \\
\hline Monocytes count & $0.7 \uparrow$ & 0.35 & 0.34 & 0.31 & 0.29 \\
\hline Eosinophils count & $0 \downarrow$ & 0.12 & 0.14 & 0.13 & 0.1 \\
\hline Basophils count & 0 & 0.03 & 0.05 & 0.02 & 0.04 \\
\hline 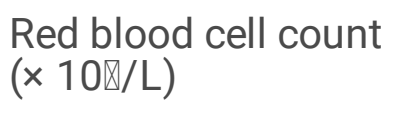 & 4.37 & 4.98 & 5.09 & 5.12 & 4.49 \\
\hline $\begin{array}{l}\text { Platelet } \\
\text { count }(\times 10 \otimes / L)\end{array}$ & 170 & 167 & 235 & 212 & 213 \\
\hline $\begin{array}{l}\text { Mean platelet } \\
\text { volume(f1) }\end{array}$ & 9.1 & $8.8 \downarrow$ & $8.6 \downarrow$ & $8 \downarrow$ & $7.4 \downarrow$ \\
\hline Creatinine $(\mu \mathrm{mol} / \mathrm{L})$ & NA & 55.4 & 68.3 & 67.9 & 42.4 \\
\hline $\begin{array}{l}\text { C-reactive } \\
\text { protein(mg/L) }\end{array}$ & NA & 1 & 2.43 & 1.22 & 6.21 \\
\hline
\end{tabular}




\begin{tabular}{|c|c|c|c|c|c|}
\hline Total protein $(\mathrm{g} / \mathrm{L})$ & NA & 68.9 & 66.6 & 66.8 & 66 \\
\hline $\operatorname{Albumin}(\mathrm{g} / \mathrm{L})$ & NA & 46.9 & 46.6 & 49.5 & 47.4 \\
\hline $\begin{array}{l}\text { Total } \\
\text { bilirubin }(\mu \mathrm{mol} / \mathrm{L})\end{array}$ & NA & 11.47 & 4.93 & 5.44 & 10.54 \\
\hline $\begin{array}{l}\text { Alanine amino } \\
\text { transferase }(\mathrm{U} / \mathrm{L})\end{array}$ & NA & 23.8 & 37.4 & 30.6 & 25.9 \\
\hline Prealbumin(mg/L) & NA & 257.6 & 276.1 & 268.9 & $154 \downarrow$ \\
\hline Prothrombin time(s) & NA & 10.5 & 10.4 & 10.1 & 10.7 \\
\hline Fibrinogen(g/L) & NA & $4.25 \uparrow$ & 2.42 & 2.7 & 3.74 \\
\hline $\begin{array}{l}\text { Activated partial } \\
\text { thromboplastin } \\
\text { time(s) }\end{array}$ & NA & 27.7 & 29.1 & 30.7 & 29.5 \\
\hline Procalcitonin(ng/ml) & NA & - & 0.036 & 0.035 & 0.03 \\
\hline Potassium(mmol/L) & NA & 3.56 & 3.87 & 4.11 & $3.48 \downarrow$ \\
\hline Sodium(mmol/L) & NA & 137.8 & $136.6 \downarrow$ & 140.4 & 138.1 \\
\hline Glucose(mmol/L) & NA & 5.02 & 4.71 & 5.17 & 5.26 \\
\hline $\begin{array}{l}\text { Aspartate amino } \\
\text { transferase }(\mathrm{U} / \mathrm{L})\end{array}$ & NA & 18.8 & 25 & 18.2 & 21 \\
\hline $\begin{array}{l}\text { Lactate } \\
\text { dehydrogenase (U/L) }\end{array}$ & NA & 203 & 243 & 155 & 236 \\
\hline
\end{tabular}

\section{Figures}

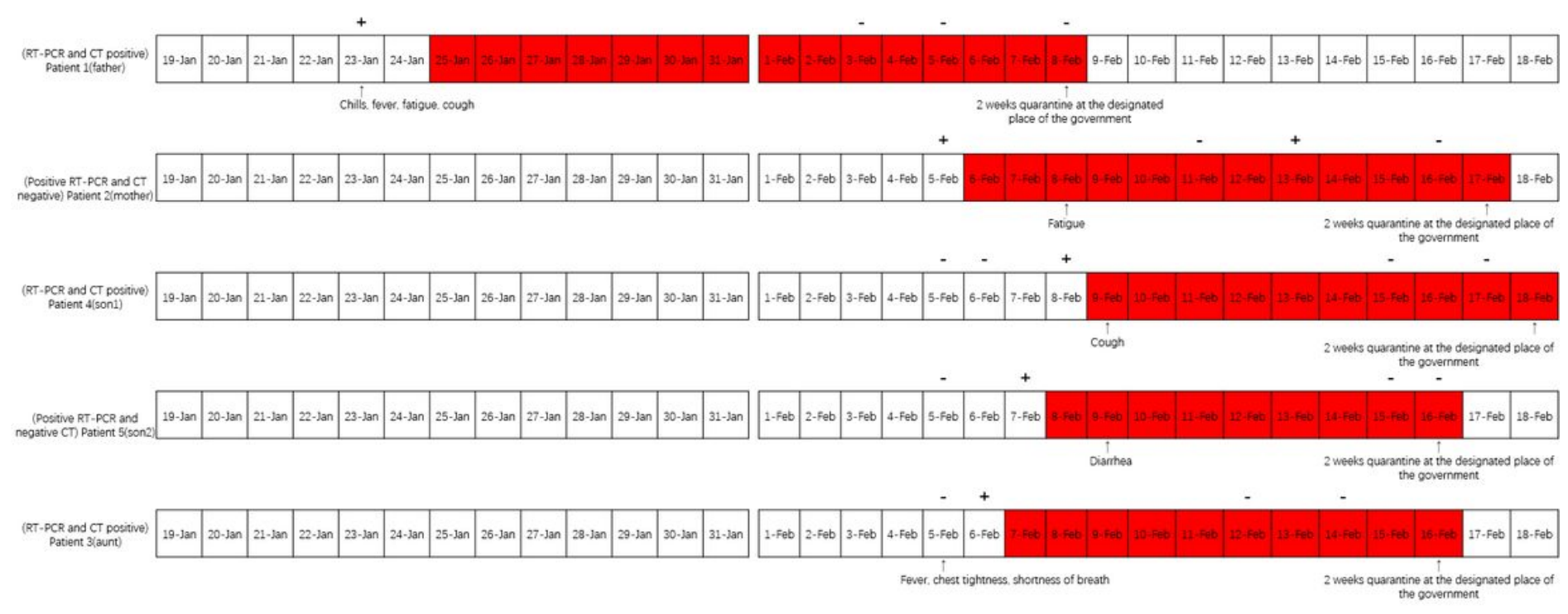

Figure 1 
Chronology of symptom onset of the Hainan family. Dates filled in red are the dates on which patients 15 are hospitalized. "+" represents the patient had positive swab test. "-" represents that the patient had negative test.
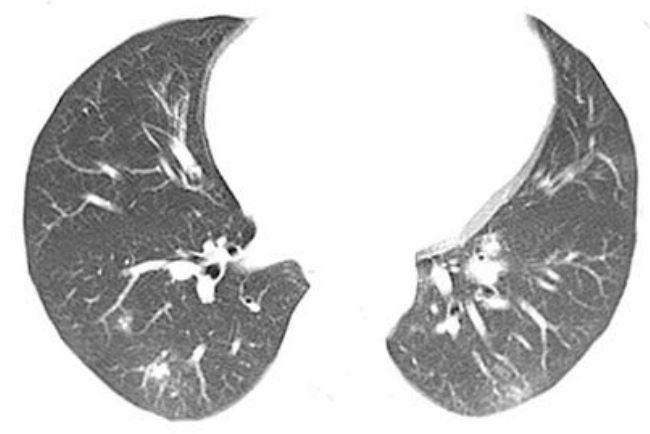

A.February 9, 2020



D.February 7, 2020

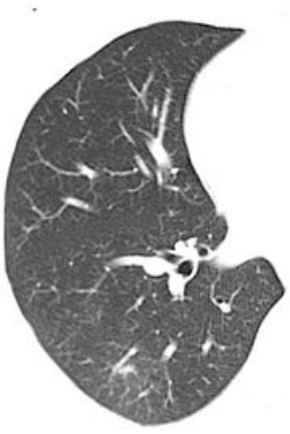

B.February 12, 2020

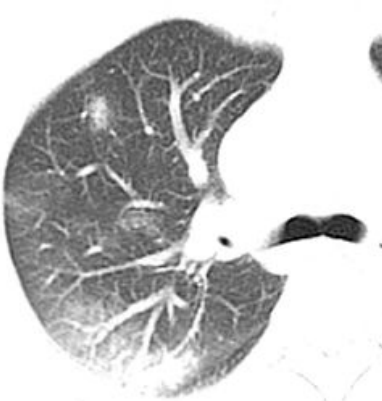

E.February 10, 2020


C.February 16, 2020
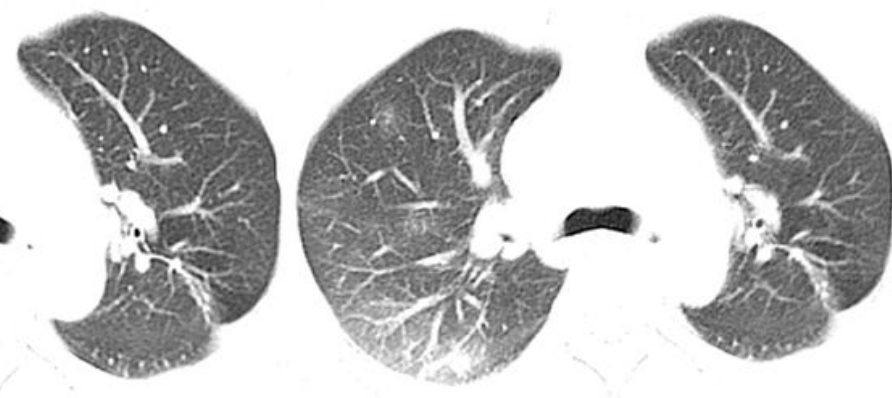

F.February 13, 2020

Figure 2

Representative images of the thoracic CT scans showing multifocal ground-glass and subsegmental areas of consolidation in the lungs of patient $3(A, B, C)$, patient $5(D, E, F)$. 